\title{
Physics and decisions: an exploration
}

\author{
Christian D. Schade ${ }^{1} \cdot$ Shyam Sunder $^{2}$ (D)
}

Received: 22 May 2020 / Accepted: 5 June 2020 / Published online: 22 July 2020

(c) The Author(s) 2020

\section{Theme and organization of the Humboldt-Kreis}

Human decision making attracts intensive study from many disciplinary perspectives including economics, neurosciences, philosophy and psychology, but mostly without direct links to the physical context of decisions. Jerome Busemeyer's (e.g., Busemeyer et al. 2006) work on quantum decision making and Haven and Khrennikov's (2013) work on quantum social science are notable exceptions. However, they purposely use quantum calculus as a toolbox to model probabilities, and do not claim to build a theoretical model to capture other, more fundamental aspects of decision making.

The physical basis of decision-making lies at the heart of the millennia-long debate over the existence of free will in philosophy, mostly to advocate determinism (such as within the 'clockwork' framework of Newtonian physics), but also to defend libertarianism as in the works of Kane (1985). Other novel proposals to link physics and decision making appear in Mousavi and Sunder (forthcoming) using classical physics, as well as in Schade (2018) which is based on the multiverse interpretation of quantum mechanics.

The Humboldt-Kreis was organized to explore the role physical laws play in understanding conscious human behavior, especially decision making. On one hand, there is the possibility of laws whose applicability transcends the traditional divide between the inanimate and animate aspects of our world. What can we learn from them about a person's decisions in isolation and in organization? On the other hand,

This paper precedes six short papers resulting from a workshop held at Humboldt-Universität zu Berlin, Berlin, Germany, the 'Humboldt-Kreis.' Those papers are discussed within this contribution.

Christian D. Schade

cds@hu-berlin.de

Shyam Sunder

shyam.sunder@yale.edu

1 Entrepreneurial and Behavioral Decision Making, Humboldt-Universität zu Berlin, Berlin, Germany

2 School of Management, Yale University, New Haven, CT, USA 
there might be features/ abilities that are specific to the domain of consciousness, and it should then be interesting to understand the consequences.

The intent behind organizing the Kreis was to explore these precepts. At this small event, we paired scholars to ask: (1) What ideas in the work of the other can better inform your own work? And (2) what aspects of your own work might be useful to the other participant/partner?

The Humboldt-Kreis took place on December 4-6, 2019 at the School of Business and Economics, Humboldt-Universität zu Berlin (in Berlin, Germany). It was co-organized by Shabnam Moussavi (Max Planck Institute for Human Development or MPIB, Berlin, Germany), Christian D. Schade (Humboldt-Universität zu Berlin, Germany) and Shyam Sunder (Yale University, USA). Seven other scholars joined the organizers, listed here in alphabetic order: Guido Bacciagaluppi (Utrecht University, The Netherlands), Andrei Khrennikov (Linnaeus University, Sweden), Laura Martignon (Ludwigsburg University, Germany), Björn Meder (MPIB, Germany), Mehdi Moussaïd (MPIB, Germany), Saras Sarasvathy (Darden School, University of Virginia, USA), and Laurianne Vagharchakian (French Behavioural Insights Unit, Interdepartmental Directorate for Public Transformation, France). Eight participants presented their perspectives, while Meder and Vagharchakian participated in the discussions. Shyam Sunder presented a paper with Mousavi and another by himself. Christian Schade presented one paper but was granted a second timeslot to extend his explanation of the role of consciousness in his version of the quantum multiverse. Finally, the six short papers submitted by Khrennikov, Bacciagaluppi, Sarasvathy, Schade, Sunder and Mousavi with Sunder form this mini-symposium.

With one exception, the papers are based on, or closely related to, the presentations at the Kreis. Saras Sarasvathy, inspired by ideas on the quantum multiverse before and during the Kreis, decided to write her reflections on the multiverse (instead of effectuation, the topic of her talk). The event ended with two parallel workshops, one led by Guido Bacciagaluppi and Christian Schade on "Is quantum theory relevant for macro domains?", and the other by Shabnam Mousavi on the topic: "Complexity (in mathematics, natural and social sciences) and measurement", with the participants split into two groups.

\section{Some reflections on the Kreis}

Most presentations where organized pairs, each 90 min or longer, followed by dynamic discussion with a panel or the whole group. The discussion remained lively, constructive and gainful for most of the participants, as evidenced by how these papers developed beyond the formal presentations prepared for the Kreis. We start by reflecting on the two presentations that were not submitted as papers (and therefore missing from this mini-symposium), move to the presentations/papers rooted in classical physics, turn to the presentations/papers rooted in quantum mechanics, and end with a sketch of Sarasvanthy's contribution on effectuation (in her presentation) and the multiverse (in her paper).

In her exciting talk, Laura Martignon presented the mathematical framework of fast-and-frugal trees that she and her co-authors developed almost two decades ago 
(Martignon et al. 2003). In the tradition of Gerd Gigerenzer and Herbert Simon, a simple heuristic can be used to provide practical and implementable guidance in many medical, management and other decisions. She presented evidence that this heuristic outperforms other simple as well as more complex models of decision making in many situations. The decision maker can use it as a quick and accurate instrument to make choices under time pressure, limited cognition and resources. In most cases, answering just a few "yes" or "no" questions leads to accurate decisions or classification.

Mehdi Moussaïd presented an analysis of crowd behavior of people in outdoor and indoor spaces, varying the shape of halls, doors, streets, etc., and using models from statistical physics. As became clear to the audience in his inspiring talk, classical physics models can help people make decisions. His statistical physicsbased mechanical modelling of aggregate crowd behavior (setting aside a rational conscious decision-maker perspective on individual humans the crowd consists of) yields accurate predictions of interventions by, say, public decision makers. Doors, obstacles in the way, the density of individuals per square meter, etc., can all be mapped into the aggregate movement of crowds (e.g., Moussaiid et al. 2016). This can help design public buildings and other spaces. His talk is closely related (and certainly fully consistent with) the ZI (zero-intelligence) approach presented by Shyam Sunder that we discuss next.

Like Mehdi Moussaïd, Shyam Sunder also aims at showing that the macro level outcomes of social phenomena, although unquestionably emerging from complex interactions among micro level actions of individual humans, do not have to share common properties. Individual behavior does not have to be fully or even boundedly rational for the aggregate level outcomes of interactions to be quite rational. Specifically, according to his work on zero-intelligence (ZI) traders (e.g., Gode and Sunder 1993), allocatively efficient market outcomes are achieved with minimal behavioral assumptions and a negligible degree of rationality.

A joint lesson from Moussaïd's and Sunder's approaches is the impressive sufficiency of scarce behavioral assumptions for emergence of highly organized and predictable outcomes at the aggregate level. Clearly, Sunder's paper, not only documents the talk, it also explains the ZI approach and its large success in more detail, and adds more depth to understanding the bridge between complexity and emergence in physics and in decision making.

Mousavi and Sunder's general framework, presented in their talk and detailed in the accompanying paper, points toward more of what classical physics has to offer. They propose using the physics principle of least action to theorize and inform oneself in the animate domains of biology and social science (that include economics and decision making). The principle of least action is a compelling example of a universal principle from physics that may help us understand certain aspects of biological and social phenomena too. Minimizing cost, effort, energy, etc., are familiar to economists as well as evolutionary biologists.

Considering the presentations and papers at the Kreis, it seems a promising avenue to explore the consequences of combining the minimum action principle and the multiverse interpretation of quantum mechanics. Sure enough, classical physics is a great approximation for what happens within each of the several realities whose 
existence the multiverse concept entails, and the principle of least action should be relevant within each one of those realities.

Guido Bacciagaluppi, a science historian and philosopher with a specialization in quantum mechanics, gave an inspiring presentation on the multiverse interpretation of quantum mechanics, with a focus on one of the most exciting problems in this context, the problem of probability - and the solution proposed to it by the Oxford school, especially Deutsch (1999) and Wallace (2012). These authors derive the Max (1926) rule of quantum mechanics from normative decision theory and its rationality principles, adapted to the framework of the multiverse. Bacciagaluppi also dealt with the problem of decoherence and connected the approach by Schade (2018) (see below) with the Oxford interpretation of the quantum multiverse. The discussion showed that whereas there are some clear similarities in some of the for$\mathrm{mal} /$ technical aspects, such as the basic idea of decohering histories, this is not true of the role of consciousness advocated by Schade. The Oxford interpretation-that Bacciagaluppi was presenting here-is a realist interpretation with consciousness passively attached to all existing realities, whereas Schade sees consciousness at the core of a 'co-creation' of reality - an idealist position. ${ }^{1}$ Bacciagaluppi's paper is a great achievement, being precise in its scientific reasoning and insightful pedagogically, e.g., in his way of explaining the decoherence principle. It also follows up on the above-mentioned discussion.

Andrei Khrennikov, a quantum physicist working in the field of financial economics, presents his own approach: quantum social science and what it implies for the processing of probability (and consequently decision making). His presentation was very informative, with two notable aspects to his approach. First, it is 'purposely agnostic' with respect to actual quantum processes that may or may not play any role in the brain/decision making of individuals. He does, however, argue that human rationality might be associated with quantum (non-Boolean) rationality, whereas humans must be judged as being irrational when applying standard expected utility theory. More pronounced in the paper than in his presentation, Khrennikov gives a brief introduction into his own interpretation of quantum mechanics, the Växjö interpretation, (related to Bohr's but going beyond it) as more convenient for applications outside physics. In this context, he also introduces his quantum-like paradigm (Khrennikov 1999). There were some interesting discussions inspired by this talk.

In his presentation, Christian Schade started with a brief introduction into a novel version of the multiverse interpretation of quantum mechanics, the clustered-minds multiverse (CMM) (monograph: Schade 2018). He also explained his concept of vectorial choices, where consciousness, based on the individual's preferences, is allocated to different realities to a different extent. By doing so, consciousness executes free will. Notwithstanding the existence of free will in principle, Schade also discussed some of the restrictions to this general possibility. During Schade's presentation, Khrennikov wanted to know what the difference is between weights-in

\footnotetext{
1 Specifically, Schade (2018, chap. 2, box. 2.1, pp. 32-33) advocates the idea of top-down decoherence, i.e., decoherence starting in consciousness, but without changing the basic idea of decohering histories.
} 
the vectorial formalism - and subjective probability. Schade as well as Bacciagaluppi argued that the coefficients in the respective vector cannot and should not be probabilities. (Simply put, do we think of free will being able to change the probabilities provided by the Max (1926) rule? Perhaps not.) In his paper, Schade firstly deals with the problem of free will and the solution offered by the CMM. Secondly, based on some interim discussions with Kathryn Laskey (George Mason University, USA) that started after the Kreis, he critically reflects on the feasibility of singularreality free will. Finally, Schade comments on the 'choice matters' concept, proposed by Sarasvathy in her written contribution to this symposium.

Indeed, a good example of the success and the value of interactions at the Humboldt-Kreis was Sarasvathy's paper as distinct from her presentation. In her presentation, she talked about the many inspiring facets of her effectuation concept, briefly relating it to the multiverse concept, based on her reflection on Schade's book on the subject, where at some point, effectuation is advertised as 'multiverse proof' (Schade 2018, chaps. 8-10). ${ }^{2}$ Participants found Sarasvathy's presentation inspiring and convincing, spanning from the original concept of effectuation (Sarasvathy 2001), over many empirical and theoretical developments, to her quick mention of potential bridges between effectuation and the multiverse. In her paper, Sarasvathy only briefly mentions effectuation, and is mainly concerned with her reflections on the multiverse perspective. So, perhaps due to the presentations by Bacciagaluppi and Schade, the discussions at the Kreis may have inspired Sarasvathy to develop her own thoughts on this new framework.

We cite Sarasvathy's paper as an example to suggest that all participants benefitted from the interdisciplinary and interactive format of the Humboldt-Kreis, perhaps not as explicitly visible in the other papers. As evidenced by the discussions during presentations, coffee breaks and meals, most participants seem to have developed their thinking in various ways. It is also reflected in the evolution from the respective presentations to papers in this mini-symposium. Therefore, the Kreis is viewed as a large success. All participants thank Christian Schade and Humboldt University for hosting it.

Acknowledgements Open Access funding provided by Projekt DEAL. We gratefully acknowledge editing help of Elizabeth Viloudaki.

Open Access This article is licensed under a Creative Commons Attribution 4.0 International License, which permits use, sharing, adaptation, distribution and reproduction in any medium or format, as long as you give appropriate credit to the original author(s) and the source, provide a link to the Creative Commons licence, and indicate if changes were made. The images or other third party material in this article are included in the article's Creative Commons licence, unless indicated otherwise in a credit line to the material. If material is not included in the article's Creative Commons licence and your intended use is not permitted by statutory regulation or exceeds the permitted use, you will need to obtain permission

\footnotetext{
2 The reason for this is that according to Schade's clustered-minds multiverse approach, allocations of consciousness are expected to be balanced within one reality (with the different versions of the individuals residing here) and unbalanced among realities (resulting from vectorial choices and the above-mentioned restrictions). Compared to most 'normative' approaches to decision making, effectuation reduces decisional conflicts (thus, in principle, also among different versions of an individual), it tends to move away from harsh tradeoffs, such as between risk and return (because of the affordable-loss principle, for instance).
} 
directly from the copyright holder. To view a copy of this licence, visit http://creativecommons.org/licen ses/by/4.0/.

\section{References}

Busemeyer JR, Wang Z, Townsend JT (2006) Quantum dynamics of human decision-making. J Math Psychol 50:220-241

Deutsch D (1999) Quantum theory of probability and decisions. Proc R Soc Lond Ser A Math Phys Eng Sci 455:3129-3137

Gode DK, Sunder S (1993) Allocative efficiency of markets with zero intelligence traders: market as a partial substitute for individual rationality. J Polit Econ 101(1):119-137

Haven E, Khrennikov A (2013) Quantum social science. Cambridge University Press, Cambridge

Kane RH (1985) Free will and values. State University of New York Press, Albany, NY

Martignon L, Vitouch O, Takezawa M, Forster M (2003) Naive and yet enlightened: from natural frequencies to fast and frugal decision trees. In: Hardman D, Macchi L (eds) Thinking: psychological perspectives on reasoning, judgement and decision making. Wiley, Chichester

Max B (1926) Zur Quantenmechanik der Stoßvorgange. Z Phys 37:863-867

Moussaïd M, Kapadia M, Thrash T, Sumner RW, Gross M, Helbing D, Hölscher C (2016) Crowd behaviour during high-stress evacuations in an immersive virtual environment. J R Soc Interface 13(122):20160414

Sarasvathy S (2001) Causation and effectuation: toward a theoretical shift from economic inevitability to entrepreneurial contingency. Acad Manag Rev 26:243-263

Schade CD (2018) Free will and consciousness in the multiverse: physics, philosophy and quantum decision making. Springer, Berlin

Wallace D (2012) The Emergent Multiverse: quantum theory according to the Everett interpretation. Oxford University Press, Oxford

Publisher's Note Springer Nature remains neutral with regard to jurisdictional claims in published maps and institutional affiliations. 